\title{
Resistivity minima and Kondo effect in ferromagnetic GaMnAs films
}

\author{
H. T. He, C. L. Yang, W. K. Ge, and J. N. Wang ${ }^{\text {a) }}$ \\ Physics Department, The Hong Kong University of Science and Technology, Clear Water Bay, \\ Kowloon, Hong Kong, China \\ X. Dai \\ Physics Department, The University of Hong Kong, Pokfulam Road, Hong Kong, China \\ Y. Q. Wang \\ Institute of Solid State Physics, Chinese Academy of Sciences, Hefei, Anhui 230031, China
}

(Received 4 April 2005; accepted 26 August 2005; published online 11 October 2005)

\begin{abstract}
The temperature dependence of the resistivity of ferromagnetic GaMnAs, as-grown or lowtemperature-annealed samples is measured from 2 to $290 \mathrm{~K}$. A resistivity minimum is observed with a corresponding temperature $T_{M}$ around $10 \mathrm{~K}$ for each sample. Below $T_{M}$, the resistivity exhibits logarithmic temperature dependence, as $\alpha \ln (T)$, and $\alpha$ is independent of the external magnetic field up to $9 \mathrm{~T}$. Such behavior is explained in terms of the Kondo effect arising from the presence of $\mathrm{Mn}$ interstitials in the GaMnAs samples. In addition, a well-defined $T$-squared dependence of resistivity is found in the temperature range between $T_{M}$ and the Curie temperature $\left(T_{C}\right)$, which is attributed to single magnon scattering. (C) 2005 American Institute of Physics.

[DOI: 10.1063/1.2108131]
\end{abstract}

The ferromagnetic GaMnAs system has been studied extensively in the past decades due to its potential applications in spintronics. ${ }^{1,2}$ As Mn atoms are doped into a GaAs lattice, most of the $\mathrm{Mn}$ atoms occupy Ga sites $\left(\mathrm{Mn}_{\mathrm{Ga}}\right)$. These $\mathrm{Mn}_{\mathrm{Ga}}$ act as single acceptors and contribute local spins due to the half-filled $3 d$ shells. Below the Curie temperature $\left(T_{C}\right)$, a hole-mediated ferromagnetic coupling among these local spins is established. ${ }^{3,4}$ But besides $\mathrm{Mn}_{\mathrm{Ga}}$, there is also a certain amount of $\mathrm{Mn}$ interstitials $\left(\mathrm{Mn}_{I}\right)$ present in the lattice. They are double donors and tend to form antiferromagnetically coupled $\mathrm{Mn}_{\mathrm{Ga}}-\mathrm{Mn}_{I}$ pairs, which strongly suppress the established ferromagnetism. ${ }^{5,7}$ It has been shown that lowtemperature annealing can reduce the $\mathrm{Mn}_{I}$ concentration and improve $T_{C}$ significantly. ${ }^{5,9,10}$ In the present work, for the first time, we show that these $\mathrm{Mn}_{I}$ play key roles in the low-temperature transport behavior of a GaMnAs system, manifested by a Kondo-related resistivity minimum $\left(\rho_{M}\right)$ observed at a low temperature $T_{M}$. A logarithmic temperature dependence of the resistivity is observed below $T_{M}$ for both the as-grown and low-temperature-annealed ferromagnetic GaMnAs films. Such temperature dependence is found to be independent of the applied magnetic field. These phenomena can be well understood based on an analysis of the effectivefield distribution present at each Mn-ion site, which takes into account the antiferromagnetic interaction of $\mathrm{Mn}_{\mathrm{Ga}}-\mathrm{Mn}_{I}$ pairs as well as the hole-mediated ferromagnetism. In addition, a well-defined $T^{2}$ dependence of the resistivity is also observed between $T_{C}$ and $T_{M}$, which is ascribed to single magnon scattering.

A $30 \mathrm{~nm}$ GaMnAs film with $5.2 \%$ Mn composition was grown by low-temperature molecular-beam epitaxy (MBE) in a Riber $32 \mathrm{MBE}$ system on (100) semi-insulating GaAs substrate. Hall-bar structures were fabricated using standard photolithography techniques. Low-temperature annealing was carried out in $\mathrm{N}_{2}$ gas. The resistivity at different tem-

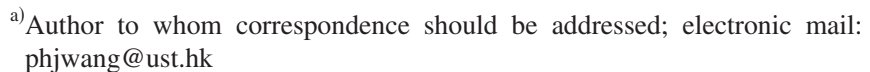

peratures and under various magnetic fields was measured using a standard four-probe technique. Four samples were investigated, and they are labeled A, B, C, and D, corresponding to the as-grown sample and the samples annealed at 160,200 , and $260{ }^{\circ} \mathrm{C}$ for $2 \mathrm{~h}$, respectively.

Figure 1 shows the temperature dependence of the resistivity $[\rho(T)]$ from 2 to $290 \mathrm{~K}$ for samples A, B, C, and D, respectively. The Curie temperature estimated from the temperature at the peak resistivity ${ }^{9,10}$ is indicated for each sample. In comparison with the as-grown sample A, the three annealed samples exhibit lower resistivity and higher $T_{C}$ with increasing annealing temperatures, which is consistent with the previous results. ${ }^{9,10}$ The four samples are all metallic, evidenced by the sharp decrease of resistivity below $T_{C}$. However, the most interesting feature is that a resistivity minimum $\rho_{M}$ at a low temperature $T_{M}$ is observed around $10 \mathrm{~K}$ for each sample. Detailed analysis reveals that the re-

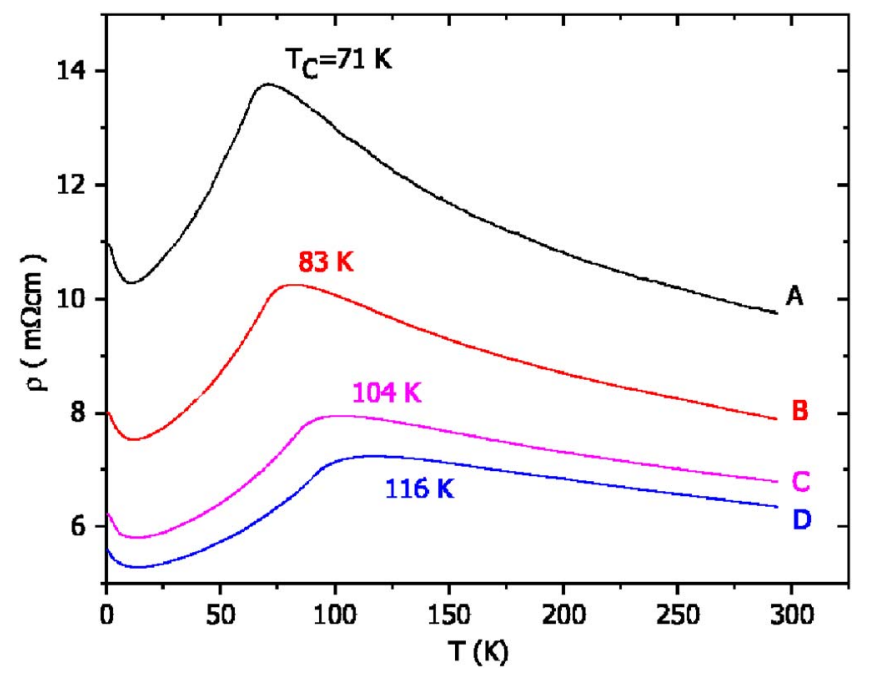

FIG. 1. (Color online) The temperature dependence of the resistivity $\rho(T)$ for samples A, B, C, and D, with the corresponding $T_{C}$ indicated. 


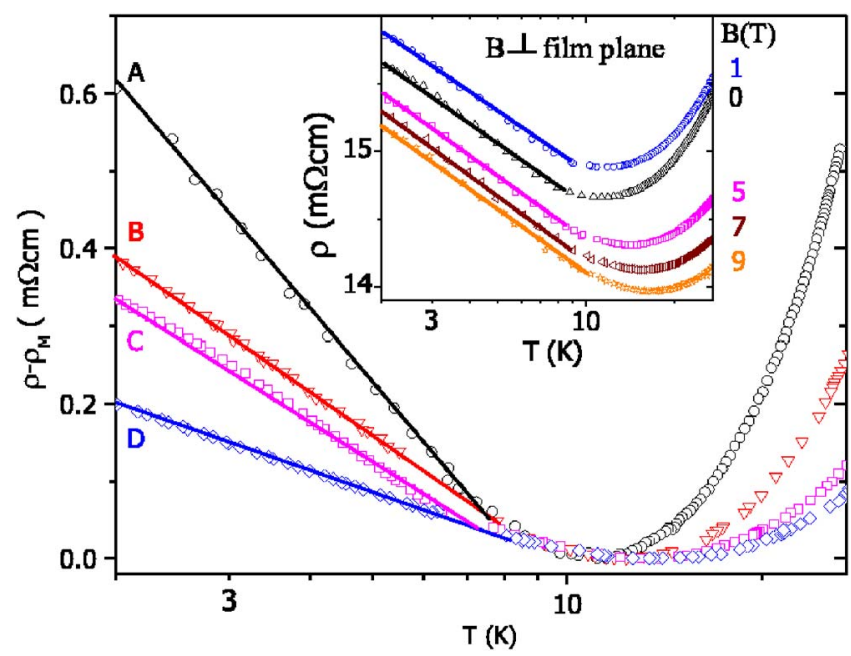

FIG. 2. (Color online) The $\rho$ vs $\ln (T)$ curve at low temperatures for each sample shows a logarithmic temperature dependence of the resistivity. The resistivity is normalized by subtracting $\rho_{M}$. The inset shows $\rho(T)$ curves for sample A under the magnetic fields indicated. The magnetic field $B$ is applied perpendicularly to the sample film. Lines are guides for the eyes.

sistivity below $T_{M}$ exhibits logarithmic temperature dependence, i.e., $\alpha \ln (T)$, as illustrated clearly in $\rho$ vs $\ln (T)$ plots in Fig. 2. For clarity, the resistivity of each sample has been normalized by subtracting the minimum resistivity $\rho_{M}$. It is also evident that $\alpha$, the slope of each curve below $T_{M}$, decreases with an increase of annealing temperature (or $T_{C}$ ).

We attribute the above-mentioned $\ln (T)$ behavior to the Kondo effect, ${ }^{11,12}$ which was first observed in dilute magnetic alloys and is known to give rise to a logarithmic temperature dependence of resistivity at low temperature. It is well known that the Kondo effect is due to the presence of some free local spins in the system. In a ferromagnetic GaMnAs system it has been shown that the antiferromagnetic superexchange from the $\mathrm{Mn}_{\mathrm{Ga}}-\mathrm{Mn}_{I}$ pairs coexists with the well-known hole-mediated ferromagnetism. ${ }^{5,7}$ It is therefore not surprising that some local spins may stay at zero internal magnetic field.

This situation is analogous to that in certain amorphous ferromagnets. ${ }^{13,14}$ The Kondo contribution to the resistivity at low temperatures in such amorphous ferromagnets is associated with the effective-field distribution of magnetic ions, expressed in terms of $P(H) .{ }^{14} P(H)$ represents the probability of a spin staying at a local internal magnetic field $H$. In a GaMnAs system, if all Mn atoms occupied the Ga sites and were ferromagnetically coupled via hole mediations, the corresponding $P(H)$ would only exhibit a peak located at high positive $H$, as shown by the dashed curve in Fig. 3. As

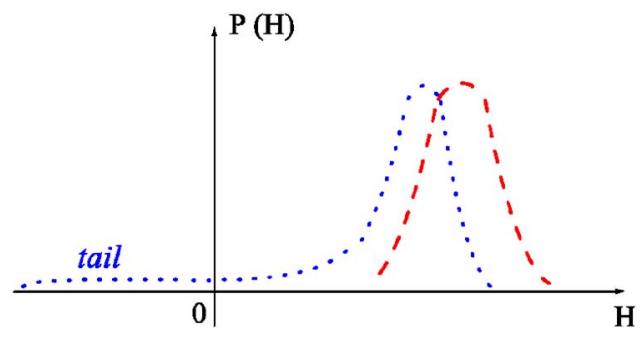

FIG. 3. (Color online) Schematic plots of the effective-field distribution $P(H)$ in a ferromagnetic system when both the antiferromagnetic superexchange interaction and the dominant ferromagnetism are considered (dotted line) and only the ferromagnetic interaction is considered (dashed line).

Downloaded 22 Feb 2007 to 143.89 .106 .83 . Redistribution subjed a result, there is no free spin present in the lattice and the Kondo effect is not expected.

However, it is well established that Mn interstitials are present in the GaMnAs system and form $\mathrm{Mn}_{\mathrm{Ga}}-\mathrm{Mn}_{I}$ pairs due to electrostatic attraction. The spins in these pairs are coupled by the antiferromagnetic superexchange interaction. The calculated strength of this superexchange is $71 \mathrm{~K}^{7}$ When the antiferromagnetic superexchange interactions from these pairs are taken into account, together with the holemediated ferromagnetism, $P(H)$ is expected to have a long and flat tail extending to zero and even deep into negative $H$, in addition to the peak located at high positive $H$, as schematically illustrated in Fig. 3 by the dotted curve. It means that the coexistence of the antiferromagnetic superexchange interaction from these $\mathrm{Mn}_{\mathrm{Ga}}-\mathrm{Mn}_{I}$ pairs and the well-known hole-mediated ferromagnetism results in some free spins at zero field below $T_{C}$. Therefore, the Kondo effect at low temperatures is expected in the presence of $\mathrm{Mn}_{\mathrm{Ga}}-\mathrm{Mn}_{I}$ pairs.

After low-temperature annealing of the as-grown GaMnAs sample, the $\mathrm{Mn}_{I}$ concentration is reduced, ${ }^{5,6}$ the contribution of antiferromagnetic coupling from $\mathrm{Mn}_{\mathrm{Ga}}-\mathrm{Mn}_{I}$ pairs is weakened, and the hole-mediated ferromagnetism is enhanced, so the tail of $P(H)$ becomes less prominent around zero $H$, and fewer free spins are present in the lattice. Therefore, the Kondo-related scattering decreases and the Kondo contributions measured by $\alpha$ should be reduced with increase of the annealing temperatures (or $T_{C}$ ), as evident in the experimental results shown in Fig. 2.

The long flat tail extending to negative $H$ in the qualitative $P(H)$ distribution curve shown in Fig. 3 can also explain the $\rho(T)$ measurement results under external magnetic fields. The inset of Fig. 2 shows the temperature dependence of the resistivity of sample $\mathrm{A}$ at low temperatures with external magnetic fields applied perpendicular to the film plane. It clearly shows that the Kondo contribution $\alpha$ does not change with the applied magnetic fields, except for a shift of the whole $\rho(T)$ curve due to magnetoresistance effects. The same behavior is also observed when the magnetic field is applied in plane and parallel to the current. The origin of these phenomena can be well understood in terms of the effective-field distribution illustrated in Fig. 3. The application of a magnetic field merely shifts the distribution function $P(H)$ uniformly toward higher $H,{ }^{14}$ but the long flat tail extending to negative $H$ in $P(H)$ ensures that the number of free spins at zero field changes little with external field, as does the Kondo contribution. The shift of $T_{M}$ to higher temperatures with increasing magnetic field is due to the suppression of single magnon scattering (discussed in the following paragraph) under the magnetic field.

As shown in Fig. 4, a well-defined $T$-squared temperature dependence $\left(\beta T^{2}\right)$ is observed between $T_{M}$ and $T_{C}$ for all samples. We believe that this $\beta T^{2}$ dependence is due to single magnon scattering ${ }^{15}$ arising from the ferromagnetically correlated local Mn spins. As can be seen in Fig. 4, this scattering process is suppressed by low-temperature annealing since the value of $\beta$ decreases with increasing annealing temperature (or $T_{C}$ ). The underlying physics is the decrease of spin-flip scattering when stronger ferromagnetism is established for a sample with higher $T_{C}$.

In conclusion, the presence of the Kondo effect at low temperatures is revealed in this study of the temperature dependence of the resistivity of GaMnAs films. Our analysis to AIP license or copyright, see http://apl.aip.org/api/copyright.jsp 


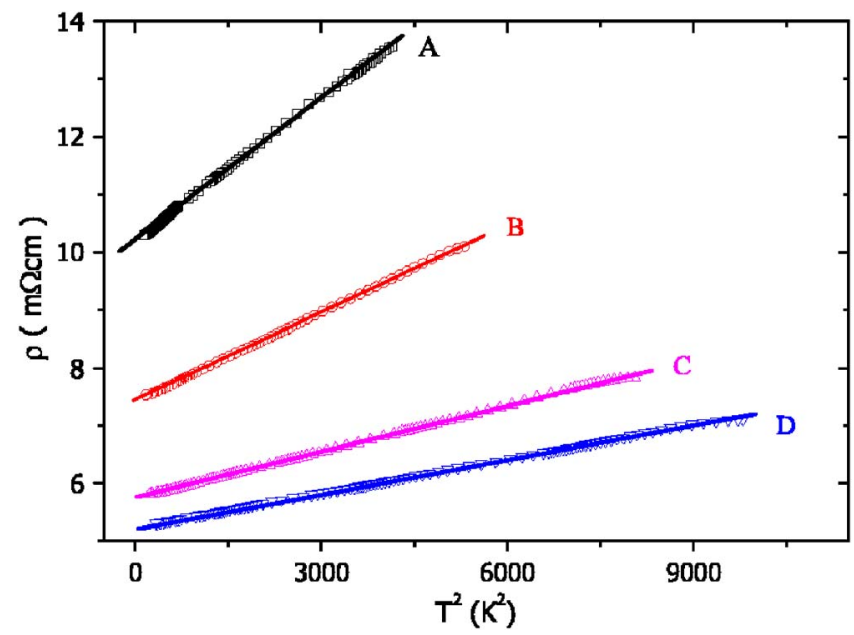

FIG. 4. (Color online) The $\rho$ vs $T^{2}$ curve for each sample between $T_{M}$ and $T_{C}$. Lines are guides for the eyes.

based on the effective-field distribution shows that the Kondo effect originates from the existence of $\mathrm{Mn}_{\mathrm{Ga}}-\mathrm{Mn}_{I}$ pairs, which are antiferromagnetically coupled. The lowtemperature annealing of GaMnAs samples suppresses the Kondo contribution by breaking the $\mathrm{Mn}_{\mathrm{Ga}}-\mathrm{Mn}_{I}$ pairs and reducing the $\mathrm{Mn}_{I}$ concentration. This demonstrates that the Mn interstitials play important roles in the low-temperature transport properties of GaMnAs films. In addition, the $T^{2}$ dependence of the resistivity between $T_{C}$ and $T_{M}$ is attributed to single magnon scattering.
This work is supported by the Research Grant Council of Hong Kong SAR, via Grant No. 603704.

${ }^{1}$ G. A. Prinz, Science 282, 1660 (1998).

${ }^{2}$ S. A. Wolf, D. D. Awschalom, R. A. Buhrman, J. M. Daughton, S. von Molnár, M. L. Roukes, A. Y. Chtchelkanova, and D. M. Treger, Science 294, 1488 (2001).

${ }^{3}$ T. Dietl, H. Ohno, F. Matsukura, J. Cibert, and D. Ferrand, Science 287, 1019 (2000).

${ }^{4}$ F. Matsukura, H. Ohno, A. Shen, and Y. Sugawara, Phys. Rev. B 57, R2037 (1998).

${ }^{5}$ K. M. Yu, W. Walukiewicz, T. Wojtowicz, I. Kuryliszyn, X. Liu, Y. Sasaki, and J. K. Furdyna, Phys. Rev. B 65, R201303 (2002).

${ }^{6}$ K. W. Edmonds, P. Boguslawski, K. Y. Wang, R. P. Campion, S. N. Novikov, N. R. S. Farley, B. L. Gallagher, C. T. Foxon, M. Sawicki, T. Dietl, M. Buongiorno Nardelli, and J. Bernholc, Phys. Rev. Lett. 92, 037201 (2004).

${ }^{7}$ J. Blinowski and P. Kacman, Phys. Rev. B 67, R121204 (2003).

${ }^{8}$ K. W. Edmonds, N. R. S. Farley, T. K. Johal, G. van der Laan, R. P. Campion, B. L. Gallagher, and C. T. Foxon, Phys. Rev. B 71, 064418 (2005).

${ }^{9}$ T. Hayashi, Y. Hashimoto, S. Katsumoto, and Y. Iye, Appl. Phys. Lett. 78, 1691 (2001).

${ }^{10}$ K. W. Edmonds, K. Y. Wang, R. P. Campion, A. C. Neumann, N. R. S. Farley, B. L. Gallagher, and C. T. Foxon, Appl. Phys. Lett. 81, 4991 (2002).

${ }^{11}$ J. Kondo, Prog. Theor. Phys. 32, 37 (1964).

${ }^{12}$ M. Daybell, in Magnetism, edited by G. T. Rado and H. Suhl (Academic, New York, 1973), Vol. 5.

${ }^{13}$ R. Hasegawa and C. C. Tsuei, Phys. Rev. B 3, 214 (1971).

${ }^{14}$ G. S. Grest and S. R. Nagel, Phys. Rev. B 19, 3571 (1979).

${ }^{15}$ I. Mannari, Prog. Theor. Phys. 22, 335 (1959). 